\title{
Modular Structure of the Local Algebras Associated with the Free Massless Scalar Field Theory
}

\author{
Peter D. Hislop ${ }^{1 \star}$ and Roberto Longo ${ }^{2 \star \star}$ \\ 1 Department of Physics, University of California, Berkeley, CA 94720, USA \\ 2 Istituto Matematico "G. Castelnuovo", Università di Roma, I-00185 Roma, Italia
}

\begin{abstract}
The modular structure of the von Neumann algebra of local observables associated with a double cone in the vacuum representation of the free massless scalar field theory of any number of dimensions is described. The modular automorphism group is induced by the unitary implementation of a family of generalized fractional linear transformations on Minkowski space and is a subgroup of the conformal group. The modular conjugation operator is the anti-unitary implementation of a product of time reversal and relativistic ray inversion. The group generated by the modular conjugation operators for the local algebras associated with the family of double cone regions is the group of proper conformal transformations. A theorem is presented asserting the unitary equivalence of local algebras associated with lightcones, double cones, and wedge regions. For the double cone algebras, this provides an explicit realization of spacelike duality and establishes the known type $I I I_{1}$ factor property. It is shown that the timelike duality property of the lightcone algebras does not hold for the double cone algebras. A different definition of the von Neumann algebras associated with a region is introduced which agrees with the standard one for a lightcone or a double cone region but which allows the timelike duality property for the double cone algebras. In the case of one spatial dimension, the standard local algebras associated with the double cone regions satisfy both spacelike and timelike duality.
\end{abstract}

\section{Introduction}

In algebraic quantum field theory, one considers a family of von Neumann algebras $R(O)$ indexed by regions $O$ of Minkowski space $M$. The modular structure of a von Neumann algebra $R(O)$ consists of a one-parameter strongly continuous unitary group $\Delta^{i \lambda}, \lambda \in \mathbb{R}$, which acts as a KMS automorphism group on $R(O)$ and its commutant $R(O)^{\prime}$, and an anti-unitary involution $J_{O}$ such that $J_{O} R(O) J_{O}=R(O)^{\prime}$.

* Supported by the National Science Foundation under Grant No. PHY-79-23251

$\star \star$ Supported in part by C. N. R. 
Of course, the pair $\left\{J_{O}, \Delta_{O}^{i \lambda}\right\}$ depends on the representation and in this paper we restrict ourselves to the vacuum representation of the local algebras.

It has been conjectured that algebraic duality holds for local algebras $R(O)$ associated with sufficiently regular regions $O \subset M$. This means that $R(O)^{\prime}=R\left(O^{c}\right)$, where $O^{c}$ is the causal complement of $O$. In a study of the duality property, Bisognano and Wichmann [1] investigated the modular structure of algebras associated with wedge regions $W$ in the Wightman framework. They showed that the modular group of $R(W)$ in the vacuum representation is induced by a one-parameter group of pure Lorentz transformations. More recently, Buchholz [2] proved that the modular group for the lightcone algebra $R\left(V_{+}\right)$in the free massless scalar field theory of odd spatial dimension $>1$ is induced by the global dilatation group.

In this paper, we describe the modular structure of $R(O)$ in free massless scalar field theory when $O$ is a double cone region. Bisognano and Wichmann exploited the geometrical simplicity of the wedge regions in their arguments which apply to any local quantum field theory [3]. Buchholz' result depends on the peculiar dynamics of free massless scalar field theory as is embodied in the dilatation invariance of the theory. The present study is based on the geometrically simple relationship between $O$ and $O^{c}$, when $O$ is a double cone centered at the origin, and on the conformal symmetry of the theory [4].

The work herein is motivated by the following observation. Since the fields are free, the local algebra $R(O)$ is determined by the value of the field and its time derivative on the $t=0$ hypersurface. In two dimensions, this is simply an interval, say $(-1,1)$. The Cauchy data for $R(O)$ is mapped by the modular conjugation operator to that for $R\left(O^{c}\right)=R(O)^{\prime}$. We note that the inversion map $x \rightarrow \frac{1}{x}$ sends the interval $(-1,1)$ onto $(-\infty,-1) \cup(1, \infty)$. Moreover, the interval $(-1,1)$ is preserved by the one-parameter group of coordinate transformations $x \rightarrow x^{\prime}(\lambda)$ given by $\left(x^{\prime}-1\right)\left(x^{\prime}+1\right)^{-1}=e^{\lambda}(x-1)(x+1)^{-1}, \lambda \in \mathbb{R}$. This transformation allows an analytic continuation to $\lambda=i \pi$ where it becomes $x \rightarrow x^{\prime}(i \pi)=\frac{1}{x}$. Consequently, these two point transformations on $M$ suggest candidates for the modular operators of $R(O)$. The main result of this paper is that these candidates are indeed the modular operators.

Beginning with these point transformations, the modular group and the modular conjugation operator for the double cone algebra can be constructed directly using standard techniques. We present here, however, a more geometrically based construction of these operators. It has long been realized that the relativistic ray inversion

$$
\rho:\left(\mathbf{x}, x_{o}\right) \rightarrow \rho(x)=\left[-\mathbf{x}\left(x^{2}\right)^{-1},-x_{o}\left(x^{2}\right)^{-1}\right], \quad x^{2} \equiv x_{o}^{2}-|\mathbf{x}|^{2},
$$

can be used to map a double cone $O$ onto a wedge $W$, an idea attributed to $\mathrm{V}$. Glaser. Analagously, one can use $\rho$ to map a double cone $O$ onto the forward lightcone $V_{+}$.

We verify explicitly that in the free massless scalar field theory $\rho$ induces a 
unitary operator $U_{\rho}$ on the one-particle space $\mathscr{H}$ and hence a unitary operator $\Gamma\left(U_{\rho}\right)$ on the boson Fock space $\Gamma(\mathscr{H})$. Our computations for $U_{\rho}$ extend those of Swieca and Völkel [4]. The existence of $U_{\rho}$ allows us to construct spatial isomorphisms between $R(O), R(W)$, and $R\left(V_{+}\right)$when the spatial dimension $d$ is odd and $d \geqq 1$. When $d$ is even, the field propagation is no longer determined by Huyghen's Principle [5] and consequently $R\left(V_{-}\right) \not \subset R\left(V_{+}\right)^{\prime}$. However, $U_{\rho}$ is still unitary on $\mathscr{H}$ and can be used to construct spatial isomorphisms between $R(W)$, $R\left(V_{+}\right)$, and $R(O)$.

It follows from the existence of the unitary operator $U_{\rho}$ that the modular structures of $R(O), R(W)$ and $R\left(V_{+}\right)$are equivalent. We show that the modular group $R(O)$ is implemented by a one-parameter group induced by a group of generalized fractional linear transformations and the modular conjugation is implemented by a product of the time reversal operator with $\Gamma\left(U_{\rho}\right)$. Moreover, this one-parameter unitary group is a subgroup of the conformal group ${ }^{1}$. The group generated by these unitary groups implementing the modular automorphism group for the algebras associated with the double cone regions is the connected identity component of the conformal group. Moreover, the modular conjugation operators for these algebras generate the group of proper conformal transformations.

By using the unitary operator $U_{\rho}$, the known results on the modular structure of $R\left(V_{+}\right)$can be obtained from those of $R(W)$ when $d>1$ and odd. Moreover, $U_{\rho}$ is used to obtain the modular structure of $R\left(V_{+}\right)$when $d$ is even. The unitary equivalence of $R(W)$ and $R(O)$ establishes the known type $I I I_{1}$ factor property of $R(O)$ and the unitary equivalence of $R(W)$ and $R\left(V_{+}\right)$establishes this property for $R\left(V_{+}\right)[6,7]$. A proof of the spacelike duality property [8] of $R(O)$ is also given. (The verification of the results of [8] for the $m=0$ case is given in [9].)

The notion of timelike duality for local algebras, i.e. $R(O)^{\prime}=R\left(O^{t}\right)$, where $O^{t}$ is the timelike complement of $O$, is a generalization of the duality for lightcone algebras discovered by Buchholz [2]: $R\left(V_{+}\right)^{\prime}=R\left(V_{+}^{t}\right)=R\left(V_{-}\right)$. It is shown by explicit construction that the timelike duality property does not hold for the local algebras associated with double cone regions $O: R\left(O^{t}\right) \subset R(O)^{\prime}$ properly. This construction suggests how the definition of the local algebra can be changed such that the new local algebras equal the standard ones for connected regions and such that the timelike duality property holds for the algebras associated with the double cone regions. In even spatial dimensions, the new local algebras associated with double cone regions satisfy a twisted timelike duality property.

The corresponding results for the free massless scalar field theory in two space-time dimensions are discussed in an appendix. It is shown that the double cone algebras defined in the usual way satisfy both spacelike and timelike duality.

\section{The Ray Inversion Map}

The free massless scalar field for spatial dimensions $d \geqq 2$ is an operator-valued tempered distribution satisfying $\phi[\square f]=0, f \in S\left(\mathbb{R}^{d+1}\right)$. We discuss the case $d=3$

1 For the case of a scalar field, we have a unitary representation of the universal covering group of the conformal group $S O_{e}(4,2) / Z_{2} \simeq S U(2,2) / Z_{4}$ 
explicitly which is typical of the odd $d>1$ cases. For the cases even $d \geqq 2$, an analogous calculation establishes the unitarity of $U_{\rho}$ on $\mathscr{H}$. The basic results for the modular structure of $R(O), O$ a double cone region, apply in all cases. The case $d=1$ is somewhat pathological yet similar results hold in that case (see Appendix 3).

The field operator $\phi[f], f \in S_{R}\left(\mathbb{R}^{4}\right)$ (the real Schwartz functions), is the usual Wightman field operator defined and, in this case, essentially self-adjoint on the dense domain $D_{o} \subset \Gamma(\mathscr{H})$ obtained from the action of the polynomial algebra generated by the field operators on the vacuum. $\Gamma(\mathscr{H})$ is the boson Fock space constructed from $\mathscr{H}=L^{2}\left[H_{o}, \theta\left(p_{o}\right) \delta\left(p^{2}\right) d^{4}(p)\right]$, where $H_{o} \equiv\left\{p \in \mathbb{R}^{4} \mid p_{o}>0, p^{2}=0\right\}$. For any open and non-empty region $O \subset \mathbb{R}^{4}, R(O)$ denotes the von Neumann algebra $\left\{\exp \left(i \phi[f]^{* *}\right) \mid f \in S_{R}(O)\right\}^{\prime \prime}$, where $\phi[f]^{* *}$ is the closure of the field operator and $S_{R}(O)$ denotes the real Schwartz functions with support in $O$. We use $D_{R}(O)$ to denote the subset of $S_{R}(O)$ consisting of function with compact support in $O$. The vacuum vector $\Omega$ is cyclic and separating for $R(O)$ by the Reeh-Schlieder Theorem provided $O^{c} \equiv \operatorname{Int}\left\{x \mid(x-y)^{2}<0, y \in O\right]$ is non-empty.

Let $T \equiv\left\{x \mid x^{2}>0\right\}$ and $S \equiv\left\{x \mid x^{2}<0\right\}$, the set of all strictly timelike and spacelike vectors, respectively. The relativistic ray inversion map $\rho$ is the involutive diffeomorphism of $T \cup S$ given by $\rho(x)=-x\left(x^{2}\right)^{-1}$; notice that $T$ and $S$ are $\rho$-invariant. For $f \in D(T \cup S)$, define $f_{\rho}(x)=I(x) x^{2} f[\rho(x)]=-\left(x^{2}\right)^{-3} f[\rho(x)]$, where $I(x)=-\left(x^{2}\right)^{-4}$ is the Jacobian of $\rho$. We define a linear transformation $U_{\rho}$ on a dense domain in $\mathscr{H}$ via the canonical isomorphism of the complex linear span of $\{\phi[f] \Omega \mid f \in D(T \cup S)\}$ into $\mathscr{H}$ by:

$$
U_{\rho} \phi[f] \Omega=\phi\left[f_{\rho}\right] \Omega, \quad f \in D(T \cup S) .
$$

Due to the conformal symmetry of the theory, we have

Theorem 1. $U_{\rho}$ extends to a unitary operator on $\mathscr{H}$.

The proof of this theorem is given in Sect. 5. $U_{\rho}$ induces a vacuum preserving unitary operator $\Gamma\left(U_{\rho}\right)$ on $\Gamma(\mathscr{H})$ such that $\Gamma\left(U_{\rho}\right) \phi[f] \Gamma\left(U_{\rho}\right)^{*}=\phi\left[f_{\rho}\right], f \in D(T \cup S)$, and consequently, for $O \subset T \cup S, \Gamma\left(U_{\rho}\right) R(O) \Gamma\left(U_{\rho}\right)^{*}=R[\rho(O)]$.

\section{The Modular Structure and Duality}

Denote by $O_{1}$ the open double cone of radius 1 centered at the origin; $V_{+}$and

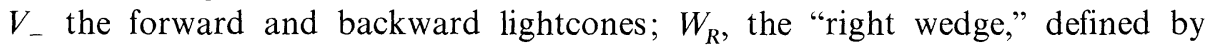
$W_{R} \equiv\left\{x\left|x_{3}>\right| x_{o} \mid\right\} ;\left\{e_{o}, e_{1}, e_{2}, e_{3}\right\}$ the canonical orthonormal basis of $\mathbb{R}^{4}$; hence $O_{1}=\left(V_{+}-e_{o}\right) \cap\left(V_{-}+e_{o}\right)$. We have

$$
\begin{aligned}
& V_{-}-\frac{1}{2} e_{o}=\rho\left(O_{1}+e_{o}\right), \\
& W_{R}+\frac{1}{2} e_{3}=\rho\left(O_{1}+e_{3}\right)
\end{aligned}
$$

by elementary computations. For example, one can use the identity

$$
[\rho(x)-\rho(y)]^{2}=x^{-2} y^{-2}(x-y)^{2}, \quad x, y \in T \cup S
$$

to show that $\rho\left(O^{t t}\right)=\rho(O)^{t t}$ if $O \subset T$ where $O^{t} \equiv \operatorname{Int}\left\{x \in \mathbb{R}^{4} \mid(x-y)^{2}>0\right.$ for all $\left.y \in O\right\}$ 
is the timelike complement of $O$. Relation (1) follows immediately because

$$
\left[\left(O_{1}+e_{o}\right) \cap \mathbb{B} e_{o}\right]^{t t}=O_{1}+e_{0} .
$$

By Theorem 1, formulas (1) and (2) and translation covariance, we have

Corollary 1. $R\left(O_{1}\right), R\left(V_{+}\right)$and $R\left(W_{R}\right)$ are spatially isomorphic. The equivalences are given by unitary operators on $\Gamma(\mathscr{H})$ induced by unitary operators on $\mathscr{H}$.

For example, $\Gamma\left[T\left(-\frac{1}{2}\right) U_{\rho} T(-1)\right]$ implements the isomorphism between $R\left(O_{1}\right)$ and $R\left(V_{+}\right)$, where $T(\lambda)$ is the one-parameter unitary group of time translations on $\mathscr{H}$.

Remark 1 . The result of $[6,7]$ and Corollary 1 provide a proof that $R\left(O_{1}\right)$ is a type $I I I_{1}$ factor [10]. Moreover, the local algebras associated with the same bounded open regions in massless and massive free scalar field theories are isomorphic [11]. These algebras are isomorphic to the local algebras in the $P(\phi)_{2}$ theory due to the local Fock character of the representation [12]. Thus, the local algebras associated with double cones in all these theories are type $I I I_{1}$ factors.

For $O=V_{+}$, the modular operators $\left\{J_{V_{+}}, \Delta_{V_{+}}^{i \lambda}\right\}$ in the vacuum representation were shown by Buchholz [2] to be given by:

$$
\Delta_{V_{+}}^{-i \lambda}=\Gamma[D(2 \pi \lambda)], \quad J_{V_{+}}=\Gamma(-\theta) .
$$

$D(\lambda)$ is the global dilatation group unitarily implemented on $\mathscr{H}$ defined by $D(\lambda) \phi[f] \Omega=\phi\left[f_{\lambda}\right] \Omega$, where $f_{\lambda}(x)=e^{-3 \lambda} f\left[\beta_{-\lambda}(x)\right], \beta_{\lambda}(x)=e^{\lambda} x, f \in S\left(\mathbb{R}^{4}\right) . \Theta$ is the anti-unitary PCT operator given by $\Theta=I_{s} I_{c} I_{t}$, the product of space reflection, charge conjugation, and time reversal operators on $\mathscr{H}\left(I_{c}\right.$ acts as the identity in this case).

Let $t(\cdot)$ denote the time translation on $\mathbb{R}^{4}$ and consider the one-parameter group $v_{\lambda}$ of conformal transformations conjugate to the group $\beta_{\lambda}$ by the transformation $\underline{t}(1) \rho t\left(\frac{1}{2}\right)$. Since $v_{\lambda}$ commutes with space rotations, its action on $\mathbb{R}^{4}$ is determined by its action on the invariant $x_{0} x_{3}$-plane where it is naturally expressed in terms of the variable $z_{1}=x_{0}+x_{3}$ and $z_{2}=x_{0}-x_{3}$. It is given by:

$$
z_{i}(\lambda)=\left\{\frac{\left(1+z_{i}\right)-e^{\lambda}\left(1-z_{i}\right)}{\left(1+z_{i}\right)+e^{\lambda}\left(1-z_{i}\right)}\right\} \quad i=1,2 ; \lambda \in \mathbb{R},
$$

generalizing the formula given in the introduction. We mention that $v_{\lambda}$ is analogously conformally conjugate to a one-parameter group of pure Lorentz transformations.

The coordinate transformation $v_{\lambda}, \lambda \in \mathbb{R}$, is not everywhere defined. For each $\lambda \in \mathbb{R}$, the map $z_{i} \rightarrow z_{i}(\lambda), i=1,2$, is singular on the lines $z_{i}=\left(1+e^{\lambda}\right)\left(e^{\lambda}-1\right)^{-1}$ which move off to infinity as $|\lambda| \rightarrow 0$ and approach $z_{i}=1$ as $\lambda \rightarrow \infty$ and $z_{i}=-1$ as $\lambda \rightarrow-\infty$. The regions $O_{1}$ and $O_{1}^{t} \cup O_{1}^{c}$ are both separately invariant under the family of coordinate transformations $v_{\lambda}, \lambda \in \mathbb{R}$. This family forms a one-parameter group of transformations on $O_{1}$ but not on $O_{1}^{t} \cup O_{1}^{c}$. The fact that $O_{1}^{c}$ is not invariant under $v_{\lambda}$ and the discontinuity of $v_{\lambda}$ on $O_{1}^{t} \cup O_{1}^{c}$ does not introduce any inherent difficulty in these considerations. This is due to the fact that in the free massless scalar field theory in odd spatial dimensions

$$
\left\{R\left(V_{+}+e_{o}\right), R\left(V_{-}-e_{o}\right)\right\}^{\prime \prime} \subset R\left(O_{1}^{c}\right) .
$$


(In even spatial dimensions, a slightly modified condition holds. See the end of Sect. 4.)

Let $V(\lambda), \lambda \in \mathbb{R}$, be the linear operator on $\mathscr{H}$ determined by

$$
\begin{aligned}
& V(\lambda) \phi[f] \Omega=\phi\left[f_{\lambda}\right] \Omega, \\
& f_{\lambda}(x)=\gamma(x ; \lambda) f\left[v_{-\lambda}(x)\right],
\end{aligned}
$$

for $f \in D\left(O_{1}\right)$. The set of functions $D\left(O_{1}\right)$ is invariant under the mapping $f \rightarrow f_{\lambda}$, $\lambda \in \mathbb{R}$, and the above relation defines $V(\lambda)$ on a dense domain in $\mathscr{H}$. The multiplier $\gamma(x ; \lambda)$ is a rotationally invariant function having value on the $x_{0} x_{3}$-plane given by

$$
\gamma\left(z_{1}, z_{2} ; \lambda\right)=2^{+6}\left[\left(1+z_{1}\right)+e^{-\lambda}\left(1-z_{1}\right)\right]^{-3}\left[\left(1-z_{2}\right)+e^{\lambda}\left(1+z_{2}\right)\right]^{-3},
$$

and it is $C^{\infty}$ for $x \in O_{1}$. Note that a formal substitution of $\lambda=i \pi$ yields $f_{i \pi}(x)=-$ $\left(x^{2}\right)^{-3} f\left[-\mathbf{x}\left(x^{2}\right)^{-1}, x_{o}\left(x^{2}\right)^{-1}\right]$, as desired.

Theorem 2. $V(\lambda), \lambda \in \mathbb{R}$, is a one-parameter strongly continuous unitary group on $\mathscr{H}$. The modular operators for $R\left(O_{1}\right)$ are:

$$
\begin{aligned}
\Delta_{O_{1}}^{-i \lambda} & =\Gamma[\mathrm{V}(2 \pi \lambda)], \quad \lambda \in \mathbb{R}, \\
J_{O_{1}} & =\Gamma\left[I_{t} U_{\rho}\right] .
\end{aligned}
$$

Proof. The algebras $R\left(O_{1}\right)$ and $R\left(V_{+}\right)$are unitarily equivalent and, as shown after Corollary 1 , the unitary operator $\Gamma\left[T(1) U_{\rho} T\left(\frac{1}{2}\right)\right]$ implements this equivalence. It follows immediately from the facts that $\Omega$ is invariant under this operator and that $\left\{\Delta_{O_{1}}^{i \lambda}, J_{O_{1}}\right\}$ are unitary and anti-unitary, respectively, that

$$
\begin{aligned}
& J_{O_{1}}=\Gamma\left[T(1) U_{\rho} T\left(\frac{1}{2}\right)\right] J_{V_{+}} \Gamma\left[T\left(-\frac{1}{2}\right) U_{\rho} T(-1)\right], \\
& \Delta_{O_{1}}^{i \lambda}=\Gamma\left[T(1) U_{\rho} T\left(\frac{1}{2}\right)\right] \Delta_{V_{+}}^{i \lambda} \Gamma\left[T\left(-\frac{1}{2}\right) U_{\rho} T(-1)\right] .
\end{aligned}
$$

Because $J_{V_{+}}=\Gamma(-\Theta)$ and $\Delta_{V_{+}}^{-i \lambda}=\Gamma[D(2 \pi \lambda)]$ and $\Gamma$ is a faithful representation of the unitaries of $B(\mathscr{H})$ into the unitaries of $B[\Gamma(\mathscr{H})]$, we only have to check that

$$
\begin{aligned}
I_{t} U_{\rho} & =-T(1) U_{\rho} T\left(\frac{1}{2}\right) \Theta T\left(-\frac{1}{2}\right) U_{\rho} T(-1), \\
V(\lambda) & =T(1) U_{\rho} T\left(\frac{1}{2}\right) D(\lambda) T\left(-\frac{1}{2}\right) U_{\rho} T(-1) .
\end{aligned}
$$

Since all of the operators involved in these expressions are induced by geometric transformations, the theorem follows by elementary computation.

Remark 2. The one-parameter family of hyperbolas of the form $x_{3}^{2}-x_{o}^{2}-$ $2 k x_{3}+1=0,|k|>1$, in $O_{1} \cap\left\{x_{o} x_{3}\right.$-plane $\}$, is invariant under the map $v_{\lambda}, \lambda \in \mathbb{R}$. In particular, the axis $\mathbb{R} e_{o} \cap O_{1}$ is invariant.

Corollary 2. The local algebra $R\left(O_{1}\right)$ satisfies spacelike duality: $R\left(O_{1}\right)^{\prime}=R\left(O_{1}^{c}\right)$.

Proof. Let $B \equiv O_{1} \cap S$; by Lemma 1 of Appendix 2 we have $R(B)=R\left(O_{1}\right)$ and therefore

$$
R\left(O_{1}\right)^{\prime}=J_{O_{1}} R\left(O_{1}\right) J_{O_{1}}=J_{O_{1}} R(B) J_{O_{1}}=R[\rho(B)]=R\left(O_{1}^{c}\right)
$$

where we have used $\Gamma\left(I_{t}\right) R(B) \Gamma\left(I_{t}\right)=R(B)$. 
The Haag spacelike duality property for sufficiently regular regions in free massive scalar field theory was first proved in [13].

Remark 3. It follows from Theorem 2 that the modular group for $R\left(O_{1}\right)$ is a subgroup of the conformal group. The generator is a linear combination of the time translation generator and the generator of the special conformal transformation in the time coordinate. These are the only two generators of the conformal group which commute with the generators of the rotation group, which is also an automorphism group of $R\left(O_{1}\right)$. It follows from this that the group generated by all $U(\Lambda, a) \Delta_{O_{1}}^{i \lambda} U(\Lambda, a)^{-1},\{\Lambda, a\} \in P_{+}^{\dagger}$, is the identity component of the conformal group. Additionally, an examination of the infinitesimal properties of the group generated by all $U(\Lambda, a) J_{O_{1}} U(\Lambda, a)^{-1},\{\Lambda, a\} \in P_{+}^{\uparrow}$, reveals that this group contains connected components of the conformal group. Specifically, the group is generated by the identity component of the conformal group and the PCT operator, i.e. it is the group of all proper conformal transformations.

Remark 4. The Lorentz invariant distributions $D_{o}$ and $D_{1}$ [14] are separately covariant with respect to the coordinate transformation $v_{\lambda}$. This follows from Remark 3 and the conformal symmetry of the free massless field theory. A geometric proof of this fact is given, for $d>1$ and odd, in Appendix B of [15]. Slight modification of this argument establishes this for the even $d$ cases and $d=1$.

Remark 5. D. Buchholz [16] has pointed out to us the known formulas for the unitary $U_{\rho}$ in the case of the free electromagnetic field [17], namely

$$
\Gamma\left(U_{\rho}\right) F_{\mu \nu}(x) \Gamma\left(U_{\rho}\right)=\left(x^{2}\right)^{-2} S_{\mu}^{\mu^{\prime}}(x) S_{v}^{v^{\prime}}(x) F_{\mu^{\prime} v^{\prime}}(\rho(x)),
$$

where $S_{\mu v}(x)=2\left(x_{\mu} x_{v} / x^{2}\right)-g_{\mu v}$. The field strength tensor $F_{\mu v}(x)$ is defined by its two-point function

$$
\left(F_{\mu \nu}\left(\theta^{\mu v}\right) \Omega, F_{\sigma \rho}\left(\eta^{\sigma \rho}\right) \Omega\right)=\frac{2}{(2 \pi)^{3}} \int_{(\infty)} \frac{d^{3}(\mathbf{k})}{|\mathbf{k}|} \overline{\widetilde{\theta}^{\mu \nu}}(|\mathbf{k}|, \mathbf{k}) \tilde{\eta}^{\sigma \rho}(|\mathbf{k}|, \mathbf{k}) \cdot g_{\mu \rho} k_{v} k_{\sigma},
$$

where $\theta$ and $\eta$ are elements of the test function space of antisymmetric tensors. (See $[9,22]$ for a discussion of duality for the free electromagnetic field.)

\section{Timelike Duality}

Buchholz [2] established the timelike duality property $R\left(V_{+}\right)^{\prime}=R\left(V_{-}\right)$for the algebras associated with lightcones in the free massless scalar field theory of odd spatial dimension $>1$. This timelike duality property does not extend to the local algebras $R(O)$ associated with bounded double cone regions $O$, i.e. $R\left(O_{1}^{t}\right) \subset R\left(O_{1}\right)^{\prime}$, properly [16]. To see this, we introduce a family of von Neumann algebras $\bar{R}(O)$ which reduce to $R(O)$ for suitably regular regions $O$. As above, let $H_{o} \equiv$ $\left\{p \in \mathbb{R}^{4} \mid p_{o}>0, p^{2}=0\right\}$ and $\mu_{o}\left[d^{4}(p)\right] \equiv \theta\left(p_{o}\right) \delta\left(p^{2}\right) d^{4}(p)$. Let $\mathscr{E}$ be the set of distributions $T \in D\left(\mathbb{R}^{4}\right)^{\prime}$ such that the Fourier transform $\widetilde{T}$ is a continuous function in a neighbourhood of $H_{o}$ and $\widetilde{T} \uparrow H_{o} \in L^{2}\left(H_{o}, \mu_{o}\right)$. The free field can be smeared with any element $T \in \mathscr{E}$. 
Definition. $\bar{R}(O)=\left\{\exp \left(i \phi[T]^{* *}\right) \mid T \in \mathscr{E} \text {, supp }(T) \subset O\right\}^{\prime \prime}$ for any non-empty, open region $O \subset M$.

Lemma 1. If $O=V_{ \pm}$or $O=O_{1}$, then $\bar{R}(O)=R(O)$.

The proof is given in Appendix 2, Lemma 2.

The assertion that $R\left(O_{1}^{t}\right) \subset R\left(O_{1}\right)^{\prime}$, properly, is equivalent to the existence of an operator $A \in R\left(V_{+}-e_{o}\right) \cap R\left(V_{-}+e_{o}\right)$ and an operator $B \in R\left(O_{1}\right)$ such that $\left[J_{O_{1}} A J_{O_{1}}, B\right] \neq 0$. This follows because $R\left(O_{1}^{t}\right)=\left\{R\left(V_{+}+e_{o}\right), R\left(V_{-}-e_{0}\right)\right\}^{\prime \prime}$ so that $R\left(O_{1}^{t}\right)^{\prime}=R\left(V_{+}-e_{o}\right) \cap R\left(V_{-}+e_{o}\right)$ due to the timelike duality for algebras associated with lightcone regions. We construct one such pair of operators as follows. Let $f(x)=\frac{\partial}{\partial x_{1}} \delta(\mathbf{x}) h\left(x_{o}\right)$ with $h \in C_{R}^{\infty}(\mathbb{R})$ such that $h\left(x_{o}\right)=+1$ if $x_{o}>1, h\left(x_{o}\right)=-1$ if $x_{o}<-1$. Let $g(x)=\frac{\partial}{\partial x_{1}} \delta(\mathbf{x}) k\left(x_{o}\right)$ with $k \in C_{R}^{\infty}(\mathbb{R}), k(0) \neq 0$ and $\operatorname{supp}(k) \subset(-1,1)$.

Both $f$ and $g$ are in $\mathscr{E}$. Note that $f \uparrow H_{o}$ is invariant if $h$ is replaced by $h \pm 1$. Consequently, $A \equiv \exp \left(i \phi[f]^{* *}\right) \in R\left(V_{+}-e_{o}\right) \cap R\left(V_{-}+e_{o}\right)$. Set $B \equiv \exp \left(i \phi[g]^{* *}\right) \in$ $R\left(O_{1}\right)$.

A simple computation shows that $J_{O_{1}} A J_{O_{1}}=\exp \left(i \phi\left[f_{\rho}\right]^{* *}\right)$ where $f_{\rho}(x)=$ $x_{o}^{2} \frac{\partial}{\partial x_{1}} \delta(\mathbf{x}) h\left(1 / x_{o}\right)$. Now $\left[J_{O_{1}} A J_{O_{1}}, B\right]=0$ if and only if $\operatorname{Im}\left(\phi\left[f_{\rho}\right] \Omega, \phi[g] \Omega\right)=0$. This is computed using formulas (A6, A7) of Appendix 1:

$$
\begin{aligned}
\operatorname{Im}\left(\phi\left[f_{\rho}\right] \Omega, \phi[g] \Omega\right) & =\frac{-1}{3 \pi} \int_{-1}^{1} d x_{o} x_{o}^{2} h\left(1 / x_{o}\right) k^{\prime \prime \prime}\left(x_{o}\right) \\
& =\frac{-1}{3 \pi}\left[\int_{0}^{1} d x_{o} x_{o}^{2} k^{\prime \prime \prime}\left(x_{o}\right)-\int_{-1}^{0} d x_{o} x_{o}^{2} k^{\prime \prime \prime}\left(x_{o}\right)\right] \\
& =\frac{4}{3 \pi} k(0) \neq 0 .
\end{aligned}
$$

An immediate consequence of this is that $R\left(O_{1} \cap T\right) \neq R\left(O_{1}\right)$ for, if it is so, then $J_{O_{1}} R\left(O_{1} \cap T\right) J_{O_{1}}=R\left(O_{1}\right)^{\prime}=\left\{R\left(V_{+}+e_{o}\right), R\left(V_{-}-e_{o}\right)\right\}^{\prime \prime}$, contradicting the above result.

The above construction indicates which operators must be added to $R\left(O_{1}^{t}\right)$ in order to generate $R\left(O_{1}\right)^{\prime}$. Let $\mathscr{E}_{T} \subset \mathscr{E}$ denote the real linear span of the distributions of the form $D^{k} \delta(\mathbf{x}) h\left(x_{o}\right), h \in C_{R}^{\infty}(\mathbb{R})$, and $D^{k} \equiv$ finite order spatial derivative.

Definition. $R_{T}(O) \equiv\left\{\exp \left(i \phi[T]^{* *}\right) \mid T \in \mathscr{E}_{T} \text {, supp }(T) \subset O\right\}^{\prime \prime}$ for any non-empty, open region $O \subset M$. It is shown in Appendix 2, Lemma 2, that $R_{T}(O)=R(O)$ for $O=O_{1}$ or $O=V_{ \pm}$.

Remark 6. Borchers [18] showed that the usual Wightman field operator smeared in the time coordinate with an element of $S(\mathbb{R})$ is a $C^{\infty}$ operator-valued function of its spatial coordinates.

For any $f \in \mathscr{E}_{T}$ with compact support, define a linear functional $f_{\rho}$ for any $g \in D\left(\mathbb{R}^{4}\right)$ by $\left\langle f_{\rho}, g\right\rangle \equiv\left\langle f, g_{\rho}^{\#}\right\rangle$ where $g_{\rho}^{\#}(x)=-x^{-2} g[\rho(x)]$, i.e. $f_{\rho}(x)=-\left(x^{2}\right)^{-3}$ $f[\rho(x)]$. This is well defined as $g_{\rho}^{\#} \in C^{\infty}\left(\mathbb{R}^{4}\right)$. 
Lemma 2. Let $f \in \mathscr{E}_{T}$ with $\operatorname{supp}(f) \subset O_{1}$. Then $f_{\rho} \in \mathscr{E}_{T}, \quad \operatorname{supp}\left(f_{\rho}\right) \subset O_{1}^{t}$, and $U_{\rho} \phi[f] \Omega=\phi\left[f_{\rho}\right] \Omega$.

Proof. A short computation shows that $f_{\rho}$ has the form of a finite sum of terms $D^{\ell} \delta(\mathbf{x}) h_{\ell}\left(x_{o}\right)$ with $h_{\ell} \in C^{\infty}, f_{\rho} \in S\left(\mathbb{R}^{4}\right)^{\prime}$, and that $f_{\rho}$ is a continuous function in a neighbourhood of $H_{o}$. If $\operatorname{supp}(h) \subset O_{1} \cap \mathbb{R} e_{o}$ then $\operatorname{supp}\left(h_{\ell}\right) \subset O_{1}^{t} \cap \mathbb{R} e_{o}$ for all $\ell$. Let $B \subset T$ such that $O_{1}-B \subset T$. $D_{o}[\rho(B)]^{\sim} \equiv\{\widetilde{f} \mid f \in D[\rho(B)]\}$ is dense in $L^{2}\left(H_{o}, \mu_{o}\right)$. If $g \in D_{o}[\rho(B)]^{\sim}$ the map:

$$
g \mapsto \int_{(\infty)} \mu_{o}\left[d^{4}(p)\right] \tilde{g}_{\rho}(-p) \tilde{f}(p)=\int_{(\infty)} \mu_{o}\left[d^{4}(p)\right] \tilde{g}(-p) \tilde{f_{\rho}}(p)
$$

[where (A8) of Appendix 1 is used] defines a bounded linear functional on a dense linear manifold in $L^{2}\left(H_{o}, \mu_{o}\right)$. Hence, $\tilde{f}_{\rho} \in L^{2}\left(H_{o}, \mu_{o}\right)$ and $f_{\rho} \in \mathscr{E}_{T}$. The above calculation also establishes

$$
\left(U_{\rho} \phi[g] \Omega, \phi[f] \Omega\right)=\left(\phi[g] \Omega, \phi\left[f_{\rho}\right] \Omega\right)
$$

for any $g \in D[\rho(B)]$ so $U_{\rho} \phi[f] \Omega=\phi\left[f_{\rho}\right] \Omega$.

Theorem 3. $R\left(O_{1}\right)^{\prime}=R_{T}\left(O_{1}^{t}\right)=\bar{R}\left(O_{1}^{t}\right) \neq R\left(O_{1}^{t}\right)$.

Proof. By Lemma 2 above,

$$
R\left(O_{1}\right)^{\prime}=J_{O_{1}} R\left(O_{1}\right) J_{O_{1}}=\Gamma\left(U_{\rho}\right) R_{T}\left(O_{1}\right) \Gamma\left(U_{\rho}\right) \subset R_{T}\left(O_{1}^{t}\right) .
$$

Because $R_{T}\left(O_{1}^{t}\right) \subset \bar{R}\left(O_{1}^{t}\right) \subset R\left(O_{1}\right)^{\prime}$ (direct computation verifies the second inclusion), the theorem follows.

When the spatial dimension $d$ is even, the fields do not commute for timelike separations and the unitary operator $U_{\rho}$ does not map the real one-particle subspace into itself. Because of these two facts, the Tomita operator $J_{O_{1}}$ is slightly different. First, noting that the vacuum expectation value of the anticommutator of the fields vanishes for timelike separations, $J_{O}$, restricted to those operators localized on $O_{1} \cap T$ differs by a twisting factor $\Gamma(Z)$ defined as follows. Let $Z$ be the operator $Z f=$ if, $f \in \mathscr{H}$ and $\Gamma(Z)$ its promotion to $\Gamma(\mathscr{H})$. If $A$ is localized in $O_{1} \cap T$ then $J_{O_{1}} A J_{O_{1}} \in \Gamma(Z)\left\{R\left(V_{+}+e_{o}\right), R\left(V_{-}-e_{o}\right)\right\}^{\prime \prime} \Gamma(Z)^{*} \subset R\left(O_{1}\right)^{\prime}$. Second, $J_{O_{1}}$ restricted to operators localized in $O_{1} \cap S$ has the same geometric interpretation as in the odd spatial dimension case. However, because $U_{\rho}$ does not preserve the real one-particle subspace, a modification by $\Gamma(Z)$ is also required. Consequently, we have

Theorem 4. For even spatial dimension $d, R\left(O_{1}\right)^{\prime}=R\left(O_{1}^{c}\right)=\bar{R}\left(O_{1}^{t}\right)^{\sim}$, where $R^{\sim} \equiv$ $\Gamma(Z) R \Gamma(Z)^{*}$, and $J_{O_{1}}=\Gamma\left[Z I_{t} U_{\rho}\right]$.

Remark 7. For the notion of twisted duality, see [19].

As mentioned in the introduction, the unitarity of $U_{\rho}$ in the even $d$ case allows the construction of a spatial isomorphism between $R\left(O_{1}\right)$ and $R\left(V_{+}\right)$, i.e.

$$
R\left(V_{+}\right)=\Gamma\left[T\left(-\frac{1}{2}\right) U_{\rho} T(-1)\right] R\left(O_{1}\right) \Gamma\left[T(1) U_{\rho} T\left(\frac{1}{2}\right)\right] .
$$

Consequently, the modular operators for $R\left(V_{+}\right)$and $R\left(V_{-}\right)$can be obtained. We 
have, for example,

$$
\begin{aligned}
& J_{V_{+}}=\Gamma(Z \Theta), \\
& J_{V_{-}}=\Gamma(\Theta) \Gamma(Z \Theta) \Gamma(\Theta)=\Gamma(-Z \Theta) .
\end{aligned}
$$

Note that $R\left(V_{+}\right)^{\prime}$ contains operators which are not local with respect to any region. The modular automorphism group has a geometric interpretation analogous to the odd $d$ case and must be slightly modified as above.

\section{Proof of Theorem 1}

Let $\mathbb{R}_{*}^{3} \equiv \mathbb{R}^{3} \backslash\{0\}$ and $\rho_{o}: \mathbb{R}_{*}^{3} \rightarrow \mathbb{R}_{*}^{3}$ be the involutive diffeomorphism defined by $\rho_{o}(\mathbf{x})=\mathbf{x}|\mathbf{x}|^{-2}$. Then $\rho_{o}=\rho \mid \mathbb{R}_{*}^{3}$ with the natural identification of $\mathbb{R}^{3}$ as the time-zero hyperplane. For any $h \in D\left(\mathbb{R}_{*}^{3}\right)$, set $h_{\rho_{o}}(\mathbf{x})=I_{o}(\mathbf{x})|\mathbf{x}|^{2} h\left[\rho_{o}(\mathbf{x})\right]=|\mathbf{x}|^{-4}$ $h\left[\rho_{o}(\mathbf{x})\right]$ and $h_{\rho_{o}}^{\neq}(\mathbf{x})=|\mathbf{x}|^{2} h_{\rho_{o}}(\mathbf{x})$, where $I_{o}(\mathbf{x})=|\mathbf{x}|^{-6}$, the Jacobian of $\rho_{o}$. We denote by $\phi_{o}$ and $\pi_{o}$ the time-zero field and time-derivative field. Define a linear operator $U_{\rho_{o}}$ on the complex linear span of $\left\{\phi_{o}[f] \Omega \mid f \in D\left(\mathbb{R}_{*}^{3}\right)\right\}$ by

$$
U_{\rho_{o}} \phi_{o}[f] \Omega=\phi_{o}\left[f_{\rho_{o}}\right] \Omega .
$$

Lemma 1. $U_{\rho_{o}}$ extends to a unitary operator on $\mathscr{H}[4]$, and for $g \in D\left(\mathbb{R}_{*}^{3}\right)$, $U_{\rho_{o}} \pi_{o}[g] \Omega=\pi_{o}\left[g_{\rho_{o}}^{\neq}\right] \Omega$.

Proof. $U_{\rho_{o}}$ is densely defined since the Fourier transform of $D\left(\mathbb{R}_{*}^{3}\right)$ is dense in $L^{2}\left[\mathbb{R}^{3},|\mathbf{p}|^{-1} d^{3}(\mathbf{p})\right]$. It is clear that $U_{\rho_{o}}$ is an involution on its domain of definition and by formula (A1) of Appendix 1,

$$
\left\|U_{\rho_{o}} \phi_{o}[h] \Omega\right\|=\left\|\phi_{o}\left[h_{\rho_{o}}\right] \Omega\right\|=\left\|\phi_{o}[h] \Omega\right\|,
$$

so $U_{\rho_{o}}$ is isometric and extends uniquely to a unitary operator, also called $U_{\rho_{o}}$, on $\mathscr{H}$. For $h, k \in D\left(\mathbb{R}_{*}^{3}\right)$ and using formula (A3) of Appendix 1 and $U_{\rho_{o}}^{*}=U_{\rho_{o}}$, we have

$$
\begin{aligned}
\left(\phi_{o}[h] \Omega, U_{\rho_{o}} \pi_{o}[k] \Omega\right) & =\left(U_{\rho_{o}} \phi_{o}[h] \Omega, \pi_{o}[k] \Omega\right)=\left(\phi_{o}\left[h_{\rho_{o}}\right] \Omega, \pi_{o}[k] \Omega\right) \\
& =\left(\phi_{o}[h] \Omega, \pi_{o}\left[k_{\rho_{o}}^{\neq}\right] \Omega\right) .
\end{aligned}
$$

Lemma 2. The closure of $U_{\rho}$ defined in Sect. 2 equals $U_{\rho_{o}}$ and is unitary on $\mathscr{H}$.

Proof. Choose any $f \in D(T \cup S)$ and any bounded, open set $O_{f} \subset T \cup S$ such that $\operatorname{supp}(f) \subset O_{f}$. Choose any non-empty, open set $E \subset \mathbb{R}_{*}^{3}$ such that $O_{f}-E \equiv\{x-\mathbf{y} \mid$ $x \in O, \mathbf{y} \in E\} \subset S$. Let $N \subset \mathscr{H}$ denote the linear subspace generated by $\left\{\phi_{o}[h] \Omega\right.$, $\left.\pi_{o}[k] \Omega \mid h, k \in D(E)\right\}$. The subspace $N$ is dense in $\mathscr{H}$ (cf. $[13,20]$ ). We show that $U_{\rho}^{*} \supset\left(U_{\rho_{o}} \uparrow N\right)$ and as $\left(U_{\rho_{o}}\lceil N)^{* *}=U_{\rho_{o}}\right.$, it follows that $U_{\rho_{o}}=U_{\rho}^{* *}$. Now $E-$ $O_{f} \subset S$ so $\rho\left(O_{f}-E\right)=\rho\left(O_{f}\right)-\rho_{o}(E) \subset S$ by formula (3). For any $h, k \in D(E)$, we can use formulas (A4) and (A5) of Appendix 1 to show that

$$
\begin{aligned}
& \left(U_{\rho} \phi[f] \Omega, U_{\rho_{o}} \phi_{o}[h] \Omega\right)=\left(\phi[f] \Omega, \phi_{o}[h] \Omega\right), \\
& \left(U_{\rho} \phi[f] \Omega, U_{\rho_{o}} \pi_{o}[k] \Omega\right)=\left(\phi[f] \Omega, \pi_{o}[k] \Omega\right),
\end{aligned}
$$

since these equations only involve absolutely convergent Riemann integrals. 
Remark 8. The proof of Lemmas 1 and 2 allow direct generalization to the case of arbitrary $d>1$. In particular, the use of spacelike separated regions in the proof of Lemma 2 eliminates the $D_{o}$-term from the two-point functions for the cases when $d$ is even.

\section{Appendix 1}

We collect here the forms of the two-point functions for the free massless scalar field in $d=3$ spatial dimensions required for the proofs in the paper. For an account of the singular solutions of the wave equation see [14]. We define the free massless scalar field such that for $f, g \in S\left(\mathbb{R}^{d+1}\right)$, we have

$$
(\phi[f] \Omega, \phi[g] \Omega)=\frac{1}{(2 \pi)^{d}} \int_{(\infty)} \frac{d^{d}(\mathbf{p})}{2|\mathbf{p}|} \tilde{f}(-|\mathbf{p}|,-\mathbf{p}) \tilde{g}(|\mathbf{p}|, \mathbf{p}),
$$

where the Fourier transform is defined by

$$
f(p)=\int_{(\infty)} d^{d+1}(x) e^{i x \cdot p} f(x)
$$

For the case $d=3$ used explicitly in the text, we have for $f, g \in S\left(\mathbb{R}^{3}\right)$

$$
\begin{aligned}
\left(\phi_{o}[f] \Omega, \phi_{o}[g] \Omega\right) & =\frac{1}{4 \pi^{2}} \int_{(\infty)} d^{3}(\mathbf{x}) d^{3}(\mathbf{y}) \bar{f}(\mathbf{x})|\mathbf{x}-\mathbf{y}|^{-2} g(\mathbf{y}), \\
\left(\pi_{o}[f] \Omega, \pi_{o}[g] \Omega\right) & =\frac{1}{4 \pi^{2}} \int_{(\infty)} d^{3}(\mathbf{x}) d^{3}(\mathbf{y})|\mathbf{x}-\mathbf{y}|^{-2}\left(\nabla_{x} \bar{f}\right)(\mathbf{x}) \cdot\left(\nabla_{y} g\right)(\mathbf{y}), \\
\left(\phi_{o}[f] \Omega, \pi_{o}[g] \Omega\right) & =\frac{i}{2} \int_{(\infty)} d^{3}(\mathbf{x}) \bar{f}(\mathbf{x}) g(\mathbf{x}) .
\end{aligned}
$$

The integrals in (A1) and (A2) exist as absolutely convergent multiple and repeated Riemann integrals. The integral in (A3) is absolutely convergent.

We also need the following special case. If $f \in D\left(\mathbb{R}^{4}\right)$ and $h \in D\left(\mathbb{R}^{3}\right)$ are such that $\operatorname{supp}(f)-\operatorname{supp}(h) \subset T \cup S$, then:

$$
\begin{aligned}
& \left(\phi[f] \Omega, \phi_{0}[h] \Omega\right)=-\frac{1}{4 \pi^{2}} \int_{(\infty)} d^{4}(x) d^{3}(\mathbf{y}) \bar{f}(x)(x-\mathbf{y})^{-2} h(\mathbf{y}), \\
& \left(\phi[f] \Omega, \pi_{0}[h] \Omega\right)=\frac{1}{2 \pi^{2}} \int_{(\infty)} d^{4}(x) d^{3}(\mathbf{y}) \bar{f}(x) x_{0}(x-\mathbf{y})^{-4} h(\mathbf{y}) .
\end{aligned}
$$

The integrals in (A4) and (A5) are absolutely convergent multiple and repeated Riemann integrals due to the fact that $\operatorname{supp}(f)-\operatorname{supp}(h) \subset T \cup S$.

In Sect. 4, we need the following special cases. Let $f, g \in \mathscr{E}_{T}$ with $f(x)=$ $\frac{\partial}{\partial x_{1}} \delta(\mathbf{x}) h\left(x_{o}\right), g(x)=\frac{\partial}{\partial x_{1}} \delta(\mathbf{x}) k\left(x_{o}\right)$ and $h, k \in C_{R}^{\infty}(\mathbb{R})$. Then

$$
(\phi[f] \Omega, \phi[g] \Omega)=\frac{1}{6 \pi} \int_{0}^{\infty} d p p^{3} \hat{h}(-p) \hat{k}(p),
$$

where $\hat{h}(p)=(2 \pi)^{-1 / 2} \int_{(\infty)} d x e^{-i x p} h(x)$. Using the standard techniques, (A6) can be 
expressed in coordinate space and

$$
\operatorname{Im}(\phi[f] \Omega, \phi[g] \Omega)=\frac{-1}{12 \pi}\left\langle\delta^{(3)}, h^{*} k^{\ddagger}\right\rangle
$$

with $k^{\neq}(x)=k(-x)$. If $f \in \mathscr{E}_{T}$ and $g \in D\left(\mathbb{R}^{4}\right)$ such that $\operatorname{supp}(f)-\operatorname{supp}(g) \subset T$, then

$$
(\phi[g] \Omega, \phi[f] \Omega)=\frac{1}{4 \pi^{2}} \int_{(\infty)} d^{4}(x) d^{4}(y) \bar{g}(x)(x-y)^{-2} f(x) .
$$

\section{Appendix 2}

Lemma 1. $R\left(O_{1}\right)=R\left(O_{1} \cap S\right)$.

Proof. By [13], $R\left(O_{1}\right)=R_{o}\left(B_{1}\right)$ where $B_{1} \equiv O_{1} \cap \mathbb{R}^{3}$ and $R_{o}\left(B_{1}\right)=\left\{\exp \left(i \phi_{o}[f]^{* *}\right)\right.$, $\left.\exp \left(i \pi_{o}[g]^{* *}\right) \mid f, g \in D_{R}\left(B_{1}\right)\right\}^{\prime \prime}$. Let $h_{\lambda}(\mathbf{x})=h(\lambda r)$, where $r \equiv|\mathbf{x}|, h \in D_{R}\left(B_{1}\right), h \geqq 0$, and $h(r)=1$ for $0 \leqq r \leqq \frac{1}{2}$ so that $\operatorname{supp}\left(h_{\lambda}\right)$ is contained in the open ball of radius $1 /(\lambda)$ and

$$
\frac{\partial^{k}}{\partial r^{k}}\left[1-h_{\lambda}(\mathbf{x})\right]=0 \text { for } k=0,1,2, \ldots \text { and }
$$

$0 \leqq r \leqq 1 /(2 \lambda)$. For any $g \in D\left(B_{1}\right)$, set $g_{\lambda}(\mathbf{x})=\left[1-h_{\lambda}(\mathbf{x})\right] g(\mathbf{x}) ; g_{\lambda} \in D\left(B_{1} \backslash\{0\}\right)$. For any

$\ell, k \in S\left(\mathbb{R}^{3}\right)$ define norms $\|\cdot\|_{\phi, \pi}$ by

$$
\begin{aligned}
& \|\ell\|_{\phi}^{2}=\int_{(\infty)} d^{3}(\mathbf{x}) d^{3}(\mathbf{y}) \bar{\ell}(\mathbf{x})|\mathbf{x}-\mathbf{y}|^{-2} \ell(\mathbf{y}) \\
& \|k\|_{\pi}^{2}=\int_{(\infty)} d^{3}(\mathbf{x}) d^{3}(\mathbf{y})(\nabla k)(\mathbf{x}) \cdot(\nabla k)(\mathbf{y})|\mathbf{x}-\mathbf{y}|^{-2} .
\end{aligned}
$$

Then $U_{o}\left(\ell_{\lambda}\right) \equiv \exp \left(i \phi_{o}\left[\ell_{\lambda}\right]^{* *}\right) \rightarrow U_{o}(\ell)$ strongly as $\lambda \rightarrow \infty$ if $\ell_{\lambda} \rightarrow \ell$ in the $\phi$-norm and $V_{o}\left(k_{\lambda}\right) \equiv \exp \left(i \pi_{o}\left[k_{\lambda}\right]^{* *}\right) \rightarrow V_{o}(k)$ strongly if $k_{\lambda} \rightarrow k$ in the $\pi$-norm. $g_{\lambda}$ defined above satisfies

$$
\begin{aligned}
& \left\|g-g_{\lambda}\right\|_{\phi}^{2} \leqq \frac{1}{\lambda^{4}}\|g\|_{\infty}^{2}\|h\|_{\phi}^{2}, \\
& \left\|g-g_{\lambda}\right\|_{\pi}^{2} \leqq \frac{1}{\lambda^{2}}\left\{\|g\|_{\infty}^{2}\||\nabla h|\|_{\phi}^{2}+\frac{2}{\lambda}\|g\|_{\infty}\|\nabla g\|_{\infty}(h,|\nabla h|)_{\phi}+\frac{1}{\lambda^{2}}\|\nabla g\|_{\infty}^{2}\|h\|_{\phi}^{2}\right\},
\end{aligned}
$$

and hence $g_{\lambda} \rightarrow g$ in the $\phi$ - and $\pi$-norms. Consequently,

$$
R_{o}\left(B_{1}\right)=R_{o}\left(B_{1} \backslash\{0\}\right)=R\left(O_{1} \cap S\right) .
$$

Lemma 2. If $O=V_{ \pm}$or $O=O_{1}$, then $\bar{R}(O)=R_{T}(O)=R(O)$.

Proof. First note that $R(O) \subset \bar{R}(O)$ by construction. If $O=O_{1}$ and $f \in D_{R}\left(O_{1}^{c}\right), T \in \mathscr{E}$ such that $\operatorname{supp}(T) \subset O_{1}$, then letting $A \equiv \exp \left(i \phi[f]^{* *}\right)$ and $B \equiv \exp \left(i \phi[T]^{* *}\right)$, a simple calculation shows that $[A, B]=0$. Thus, $\bar{R}\left(O_{1}\right)^{\prime} \supset R\left(O_{1}^{c}\right)$ so by duality $\bar{R}\left(O_{1}\right) \subset R\left(O_{1}\right)$. 
A similar argument using timelike duality establishes $\bar{R}\left(V_{+}\right)^{\prime} \supset R\left(V_{-}\right)$and thus $\bar{R}\left(V_{+}\right)=R\left(V_{+}\right)$. Second, $R_{T}(O) \subset \bar{R}(O)=R(O)$ is globally invariant under the modular automorphism group of $R(O)$. By a standard result of Tomita-Takesaki theory, it suffices to show that $\Omega$ is cyclic for $R_{T}(O) . \Omega$ is cyclic for $R_{T}(M)$ because if $T \in \mathscr{E}_{T}$ then $\widetilde{T}(\mathbf{p})$ is a finite linear combination of elements of the form $c p_{1}^{k_{1}} p_{2}^{k_{2}} p_{3}^{k_{3}} \widehat{h}(|\mathbf{p}|)$ $\left(k_{1}+k_{2}+k_{3}=k\right)$ and the real linear manifold $D$ generated by these functions is such that $D+i D$ is dense in $L^{2}\left(H_{o}, \mu_{o}\right)$. It then follows by an argument analogous to that used in the proof of the Reeh-Schlieder Theorem that $\Omega$ is cyclic for $R_{T}(O)$.

\section{Appendix 3}

We discuss some aspects of the local algebras in the two-dimensional free massless scalar field theory. Two additional results are obtained in this case: 1 ) the timelike duality property of the double cone algebra is satisfied with the standard definition of the algebra; 2$)$ the local algebras $R(O)$ associated with bounded, simply-connected regions $O$ with non-empty interior are all unitarily equivalent and equivalent to $R(W)$, where $W$ is a wedge region. Furthermore, the spacelike duality property for the double cone algebra $R\left(O_{1}\right)$ and the timelike duality property for the forward lightcone algebra $R\left(V_{+}\right)$is satisfied.

The construction of the theory with positive definite inner product is well known. The fields are operator-valued distributions on a restricted class of functions $S_{o}\left(\mathbb{R}^{2}\right)$ consisting of those $f \in S\left(\mathbb{R}^{2}\right)$ such that $f(0,0)=0$. (We note that this theory is equivalent to a Wightman vector field theory $\partial_{\mu} \phi$.) The local algebras are accordingly defined: $R(O)=\left\{\exp \left(i \phi[f]^{* *}\right) \mid f \in S_{o, R}(O)\right\}^{\prime \prime}$. Note that if $f, g \in S_{o, R}\left(\mathbb{R}^{2}\right)$ such that $\operatorname{supp}(f)$ is timelike separated from $\operatorname{supp}(g),[\phi[f], \phi[g]]=0$ on their common domain of definition. Following Buchholz [2], one verifies that the global dilatation group induces the modular automorphism group of $R\left(V_{+}\right)$and that $R\left(V_{+}\right)^{\prime}=R\left(V_{-}\right)$.

Theorem 5. In the two-dimensional free massless scalar field theory (as defined above), $R\left(O_{1}\right)^{\prime}=R\left(O_{1}^{c}\right)=R\left(O_{1}^{t}\right)$.

Proof. We first note that $R\left(O_{1}^{c}\right)^{\prime}=R\left(O_{1}^{t}\right)^{\prime}$ because in two space-time dimensions a solution $F \in S\left(\mathbb{R}^{2}\right)^{\prime}$ of the wave equation vanishes on $O_{1}^{c}$ if and only if $F$ vanishes on $O_{1}^{t}$ provided $F(x) \rightarrow 0$ in a spacelike and timelike direction. It is therefore sufficient to prove the first equality. The operator $J_{O_{1}}$ is constructed via the unitary $U_{\rho}$ as in Theorem 1 . In the time-zero formalism,

$$
R\left(O_{1}\right)=\left\{U_{o}(h), V_{o}(k) \mid h, k \in D_{R}([-1,1]), \hat{h}(0)=0\right\}^{\prime \prime},
$$

where $U_{o}(h) \equiv \exp \left(i \phi_{o}[h]^{* *}\right), V_{o}(k) \equiv \exp \left(i \pi_{o}[k]^{* *}\right)$, and one shows that

$$
R\left(O_{1}\right)^{\prime}=J_{O_{1}} R\left(O_{1}\right) J_{O_{1}}=\left\{U_{o}\left(h_{\rho}\right), V_{o}\left(k_{\rho}\right) \mid h, k \in D_{R}([-1,1]), \hat{h}(0)=0\right\}^{\prime \prime},
$$

where $h_{\rho}(x)=x^{-2} h(1 / x)$ and $k_{\rho}(x)=k(1 / x)$. We show that for $h$ as above $U_{o}\left(h_{\rho}\right) \in$ $R\left(O_{1}^{c}\right)$; a similar argument gives $V_{o}\left(k_{\rho}\right) \in R\left(O_{1}^{c}\right)$. We may assume that

$$
\int_{0}^{1} h(x) d x=\int_{-1}^{0} h(x) d x=0 .
$$


Let $h_{\rho}=h_{L}+h_{R}$, where $\operatorname{supp}\left(h_{L}\right) \subset(-\infty,-1)$ and $\operatorname{supp}\left(h_{R}\right) \subset(1, \infty)$. An elementary computation shows $h_{L}, h_{R} \in L^{2}(\mathbb{R}, d p /|p|)$ and $\hat{h}_{L}(0)=\hat{h}_{R}(0)=0$. We then have

$$
U_{o}\left(h_{\rho}\right)=U_{o}\left(h_{L}\right) U_{o}\left(h_{R}\right) \in \bar{R}\left(-W-e_{1}\right) V \bar{R}\left(W+e_{1}\right) .
$$

By an argument similar to the one given in Lemma $1, \bar{R}(W)=R(W)$ so $U_{o}\left(h_{\rho}\right) \in R\left(O_{1}^{c}\right)$.

Due to the simple geometry and the free dynamics of the theory, we obtain the

Proposition. The local algebras $R(O), O=V_{ \pm}, W, O_{1}$ or $O$ any bounded, open, simply-connected region with non-empty interior, in the vacuum sector of the theory considered here, are all unitarily equivalent by an element of the conformal group.

Proof. If $O$ is a region as described, the time-slice property of the theory and the result of Araki [21] establish that $R(O)=R(\hat{O})$, where $\hat{O}$ is the double-cone spanned by $O$. The rest follows as in Corollary 1 .

Acknowledgements. The authors thank S. Doplicher and E. H. Wichmann for (separately) proposing the work and for many comments. The first named author thanks H. A. Warchall and M. S. Hillery for conversations on the problem. The second named author is indebted to D. Buchholz for crucial suggestions and thanks C. D'Antoni for comments. Part of this work was carried out while R. L. was visiting the University of Pennsylvania and the University of California at Berkeley.

\section{References}

1. Bisognano, J. J., Wichmann, E. H.: On the duality condition for a Hermitian scalar field. J. Math. Phys. 16, 985-1007 (1975)

2. Buchholz, D.: On the structure of local quantum fields with non-trivial interaction. In: Proc. of the Int. Conf. on Operator Algebras, Ideals and their Application in Theoretical Physics, Baumgärtel, Lassner, Pietsch, Uhlmann, (eds.) pp. 146-153. Leipzig: Teubner Verlagsgesell schaft 1978

3. Bisognano, J. J., Wichmann, E. H.: On the duality condition for quantum fields. J. Math. Phys. 17, 303-321 (1976)

4. Swieca, J. A., Völkel, A. H.: Remarks on conformal invariance. Commun. Math. Phys. 24, 319-342 (1973)

5. Courant, R., Hilbert, D.: Methods of mathematical physics, Vol. II. New York: Interscience Publishers, pp. 668-691 1962

6. Driessler, W.: On the type of local algebras in quantum field theory. Commun. Math. Phys. 53, 295297 (1977)

7. Longo, R.: Notes on algebraic invariants for non-commutative dynamical systems. Commun. Math. Phys. 69, 195-207 (1979)

8. Araki, H.: A lattice of von Neumann algebras associated with the quantum theory of a free bose field. J. Math. Phys. 4, 1343-1362 (1963)

9. Benfatto, G., Nicolò, F.: The local von Neumann algebras for the massless scalar free field and the free electromagnetic field. J. Math. Phys. 19, 653-660 (1978)

10. Araki, H., Woods, E. J.: A classification of factors. Publ. R.I.M.S., Kyoto Univ. 4A, 51-130 (1968)

11. Eckmann, J. P., Fröhlich, J.: Unitary equivalence of local algebras in the quasifree representation. Ann. Inst. Henri Poincaré 20A, 201-209 (1974)

12. Glimm, J., Jaffe, A.: The $\lambda\left(\phi^{4}\right)_{2}$ quantum field theory without cutoffs: III. The physical vacuum. Acta Math. (Uppsala) 125, 203-261 (1970)

13. Araki, H.: Von Neumann algebras of local observables for free scalar field. J. Math. Phys. 5, 1-13 (1964) 
14. Vladimirov, V. S.: Les fonctions de plusieurs variables complexes et leur application à la théorie quantique des champs, Lagowski, N. (tr.) Paris: Dunod 1967

15. Hortaçsu, M., Seiler, R., Schroer, B.: Conformal symmetry and reverberations. Phys. Rev. 5D, 25192534 (1972)

16. D. Buchholz: private communication

17. Gross, L.: Norm invariance of mass-zero equations under the conformal group. J. Math. Phys. 5, 687-695 (1964)

18. Borchers, H. J.: Field operators as $C^{\infty}$ functions in spacelike directions. Nuovo Cimento 33, 16001613 (1964)

19. Doplicher, S., Haag, R., Roberts, J. E.: Fields, observables and gauge transformations I. Commun. Math. Phys. 13, 1-23 (1969)

20. Rieffel, M. A.: A commutation theorem and duality for free bose fields. Commun. Math. Phys. 39, 153-164 (1974)

21. Araki, H.: "A generalization of Borchers theorem. Helv. Phys. Acta 36, 132-139 (1963)

22. Leyland, P., Roberts, J. E., Testard, D.: Duality for quantum free fields. Marseille Preprint 1978

Communicated by R. Haag

Received August 21, 1981; in revised form October 29, 1981 
